\title{
Towards a structural biology at organism relevant temperature and chemical conditions
}

\author{
John Richard Helliwell \\ University of Manchester, Manchester, United Kingdom; \\ john.helliwell@manchester.ac.uk
}

The three probes of the structure of matter in biology (X-rays, neutrons and electrons) have complementary properties and strengths. The balance between these three structural research probes, within their strengths and weaknesses, is perceived to change, even dramatically so at times. Of course for understanding biological systems the required perspectives are:- physiologically relevant temperatures and relevant chemical conditions. These remain very tough challenges because e.g. cryoEM looks never to set foot in room temperature and crystallization often requires non-physiological chemical conditions. X-ray crystallography especially from the synchrotron has brought huge improvements in analytical capability and dominates the PDB. CryoEM has also brought great advantage for structural studies of non-crystallisable complexes. Overall, integrated structural biology techniques and functional assays make a package towards physiological relevance of any given study. X-ray laser serial fsec crystallography experiments aimed at structural dynamics and neutron macromolecular crystallography aimed at determining protonation states of ionisable amino acids are both, as a spin off, yielding room temperature structures, as well as being damage free. Comparisons between room and cryo biological structures are increasing as the X-ray laser and neutron facilities expand in number and grow in capability; structural differences are being increasingly described in many papers. We need to expand these facility provisions for room temperature studies. Likewise the extremely bright sources such as ESRF2 ie "EBS" will bring a larger number of room temperature results through the serial crystallography approach but with X-ray radiation damage effects yet to be quantified.

Keywords: Organism relevant temperature; Organism chemistry; Extremely Bright Sources; XFELs; Neutrons; Electrons 